\title{
EL TEMA DE LAS EFECTIVIDADES EN EL CASO CONCERNIENTE A LA SOBERANÍA SOBRE PULAU LIGITAN Y PULAU SIPADAN ENTRE INDONESIA Y MALASIA*
}

\author{
Mauricio Iván del TORO HUERTA**
}

RESUMEN: El presente texto es un comentario general sobre la sentencia dictada por la Corte Internacional de Justicia, el 17 de diciembre de 2002, en el caso concerniente a la soberanía sobre Pulau Ligitan y Pulau Sipadan entre Indonesia y Malasia. En su resolución, la corte, después de considerar los diferentes títulos hechos valer por las partes y las efectividades desplegadas por cada una de ellas, declaró que la soberanía sobre las dos pequeñas islas pertenece a Malasia. La sentencia es una confirmación de los criterios sostenidos por el tribunal respecto del papel de las efectividades en las controversias de atribución territorial. Por esto, es un buen ejemplo del Estado actual de la jurisprudencia en este campo y expresa claramente la función sustitutoria de las efectividades a falta de otros títulos jurídicos.

ABSTRACT: The present text is a general comment on the decision dictated by the International Court of Justice in the case concerning sovereignty over Pulau Ligitan and Pulau Sipadan between Indonesia and Malaysia. In the judgment of 17 December 2002, the Court after valuing the different titles argued by the parts and the effectiveness deployed by each one of them, declared that the sovereignty over the two small islands belongs to Malaysia. The judgment is a confirmation of the jurisprudence sustained by the tribunal recognizing the paper of the effectiveness in the territorial controversies of attribution and it is a good example of the current state of the jurisprudence in that field. It also expresses the substituting function of the effectiveness in absence of other territorial title.

RÉSUMÉ: Le présent texte est un commentaire général sur l'affaire relative á la souveraineté sur Pulau Ligitan et Pulau Sipadan entre l'Indonésie et la Malaisie. Dans l'arrêt de 17 décembre 2002, la Cour après avoir évalué les titres différents discutés par les parties et les effectivités déployées par chacun, a déclaré que la souveraineté sur les deux petites îles appartient à Malaisie. L'arrêt est une confirmation de la jurisprudence soutenue par le tribunal qui reconnaît le papier des effectivités dans les controverses territoriales d'attribution et c'est un bon exemple de l'état courant de la jurisprudence dans ce champ. Il exprime aussi la fonction supplétif des effectivités dans absence d'autre titre territorial.

* Comentarios a la sentencia de la CIJ de 17 de diciembre de 2002.

** Facultad de Derecho de la UNAM. Agradezco el apoyo del Consejo Nacional de Ciencia y Tecnología. 
SUMARIO: I. A modo de introducción. II. El planteamiento de la controversia y los títulos jurídicos alegados por las partes. III. Esbozo sobre las relaciones entre los títulos jurídicos $y$ las efectividades. IV. Breve referencia a las efectividades $y$ la cuestión de la fecha crítica. V. El juego de las efectividades en acción. VI. Comentario final.

\section{A MODO DE INTRODUCCIÓN}

El 17 de diciembre de 2002, la Corte Internacional de Justicia (CIJ) resolvió el fondo de la controversia entre Indonesia y Malasia relativa a la soberanía sobre las islas Pulau Ligitan y Pulau Sipadan. El asunto fue sometido al tribunal mediante acuerdo especial presentado ante la Secretaría de la Corte el 2 de noviembre de 1998. En dicho acuerdo, firmado entre las partes en Kuala Lumpur el 31 de mayo de 1997 y en vigor el 14 de mayo del año siguiente, las partes solicitaron a la corte que determinara sobre la base de los tratados, acuerdos y demás evidencia aportada por las mismas si la soberanía sobre las islas Pulau Ligitan y Pulau Sipadan pertenecían a la República de Indonesia o a Malasia. En su fallo, la corte, después de valorar los diferentes títulos hechos valer por las partes y las efectividades desplegadas por cada una de ellas, decidió o declaró, por 16 votos contra uno, que la soberanía sobre las dos pequeñas islas situadas en el mar de Celebes, al noreste de la isla de Borneo, pertenece a Malasia. ${ }^{1}$

1 CIJ, Case Concerning Sovereignty over Pulau Ligitan y Pulau Sipadan, 17 de diciembre de 2002, núm. 102. www.icj-cij.org. Sobre esta sentencia véase también: Caron, David (ed.), "Internacional Decisions", en particular Colson, David A., "Sovereignty over Palau Ligitan and Palau Sipadan (Indonesia-Malasia)", American Journal of International Law, vol. 97, núm. 2, abril de 2003, pp. 398-406; Spadi, Fabio, "Pulau Ligitan and Pulau Sipidam: New Parameters for the Concept of Dependency in the Maritime Enviroment? The ICJ Judgment of 17 December 2002", en The International Journal of Marine and Coastal Law, Kluwer Law International, vol. 18, núm. 2, 2003, pp. 295-310; Merrills, J. G., "Sovereignty over Pulau Ligitan and Pulau Sipidam (Indonesia v. Malaysia), Merits. Judgment of 17 december 2002", International and Comparative Law Quarterly, vol, 52, part. 3, julio de 2003, pp. 797-802; Perrin, Delphine, "Titre Conventionnel et Effectivités: L'Affaire de la Souveraineté sur Pulau Litigan et Pulau Sipadan (Indonésie-Malaisie)", Annuaire Français de Droit International, París, CNRS Éditions, XLVIII-2002, pp. 322-342. Sobre la controversia territorial Swan Sik, Ko, "Asian Territorial Disputes, With Special Reference to the Islands of Sipadan and Ligitan: Succesion to Dutch and British Titles?", en Gill, Terry D. y Heere, Hwybo P. (eds.), Reflections on Principles and Practice of International Law. Essays in Honour of Leo J. Bouchez, Netherlands, Kluwer Law International, 2000, pp. 109-125. 
Para llegar a su decisión final, la corte hace una revisión de los diferentes títulos jurídicos hechos valer por las partes, y si bien ambas alegaron distintas efectividades con el objeto de confirmar sus títulos jurídicos de carácter convencional y como alternativa respecto de la posesión pacífica y continua de Ligitan y Sipadan por parte de Malasia sin la oposición de Indonesia o cualquier otro Estado, la corte se basa principalmente en las efectividades (effectivités) de manera independiente respecto de los otros títulos. ${ }^{2}$ En consecuencia, la sentencia de la CIJ es una revisión del tema de las efectividades en derecho internacional que confirma sus criterios anteriores respecto de la posesión efectiva, y se pronuncia específicamente sobre ciertas actividades como evidencia de dicha posesión. Desde esta perspectiva, con todas las reservas respecto de la distinción entre conflictos de atribución y de delimitación que ya apuntara la corte en el caso de la diferencia fronteriza entre Burkina Faso y Mali, la sentencia sigue el modelo clásico, según el cual, en los primeros las efectividades juegan un papel predominante. El siguiente comentario tiene por objeto hacer una revisión de la sentencia y destacar el tema de las efectividades en el conjunto de la jurisprudencia del tribunal en la materia, destacando también algunas consideraciones doctrinales sobre la relación entre el título jurídico y las efectividades.

\section{EL PLANTEAMIENTO DE LA CONTROVERSIA Y LOS TÍTULOS JURÍDICOS ALEGADOS POR LAS PARTES}

En su sentencia, la CIJ después de recordar los antecedentes generales de la controversia, analiza los diferentes títulos jurídicos invocados por las partes. Al respecto, Indonesia basa su pretensión de soberanía principalmente en la Convención de 1891 entre Gran Bretaña y Holanda, en tanto legítima sucesora de esta última; tal convenio, en su concepto, establecía el paralelo de latitud $4^{\circ}-10^{\prime}$ como la línea divisoria entre las posesiones británicas y holandesas en el aérea en el que están situadas las islas Ligitan y Sipadan. Como apoyo a dicho título convencional, Indonesia alega una serie de efectividades tanto de Holanda como de la propia Indonesia. Asimismo, en sus alegatos orales, Indonesia expresó de

2 En palabras de la corte: "The Court, having found that neither of the Parties has a treaty-based title to Ligitan and Sipadan..., will consider these effectivités as an independent and separate issue". CIJ, Case Concerning Sovereignty..., cit., nota anterior, párr. 127. 
forma alternativa, para el supuesto de que la corte desestimara su argumento basado en la Convención de 1891, que era legítima sucesora del sultán de Bulungan, quién ejerció originalmente la autoridad sobre las islas.

Por su parte, Malasia basa su pretensión en la transmisión del título jurídico de soberanía a través de una cadena de sucesiones legítimas a partir del sultán de Sulu como antiguo soberano. A partir de ahí habría subsecuentes transmisiones del título a España, Estados Unidos de América, Gran Bretaña en nombre del Estado de Borneo del Norte, Reino Unido de la Gran Bretaña e Irlanda del Norte y, finalmente, a Malasia. De igual formam en apoyo a sus argumentos Malasia considera que dicha cadena de instrumentos legales se ve confirmada por una serie de efectividades tanto británicas como de Malasia sobre las islas. Finalmente, Malasia argumenta que en el supuesto en que la corte concluya que las islas pertenecieron originalmente a Holanda, sus efectividades sobre las islas desplazarían cualquier título holandés al respecto. ${ }^{3}$

En la sentencia, la corte hace una revisión de los diferentes títulos jurídicos alegados por las partes, iniciando por el Convenio de 1891. Indonesia considera que de los términos del tratado, su contexto, su objeto y fin, se concluye que se establece una línea divisoria en el paralelo de latitud $4^{\circ}-10^{\prime}$ entre las respectivas posesiones de las partes respectivas, límite que incluye a las islas en controversia en tanto que "the line must be considered an allocation line: land areas, including islands located to the north of $4^{\circ} 10^{\prime} \mathrm{N}$ latitude were... considered to be British, and those lying to the south were Dutch" y, en tanto que las islas Pulau Ligitan y Pulau Sipadan están al sur de dicho paralelo "[i]t therefore follows that under the Convention title to those islands vested in The Netherlands, and now vests in Indonesia". ${ }^{4}$

En particular, las partes disienten de la interpretación del artículo IV del convenio, ${ }^{5}$ pues mientras para Indonesia la línea debe extenderse hasta abarcar las islas en disputa across la Isla de Sebitik, para Malasia el término across implica una línea "that crosses Sebatik from the west

3 CIJ, Case Concerning Sovereignty..., cit., nota 1, párrs. 32 y 33.

4 Ibidem, párr. 34.

5 El artículo IV señala: "From $4^{\circ} 10^{\prime}$ north latitude on the east coast the boundary-line shall be continued eastward along that parallel, across the Island of Sebittik: that portion of the island situated to the north of that parallel shall belong unreservedly to the British North Borneo Company, and the portion south of that parallel to the Netherlands". 
coast to the east coast and goes no further". De esta forma, para Malasia el convenio entre Gran Bretaña y Holanda sólo establece una línea fronteriza entre sus respectivas posesiones sobre las islas de Borneo y Sebatik, y en consecuencia la línea de delimitación se detiene en el punto más al este de esta última isla, sin que continué, como lo pretende Indonesia, hasta las islas Ligitan y Sipadan.

Después de interpretar la Convención de 1981 (siguiendo las normas consuetudinarias reflejadas en los artículos 31 y 32 de la Convención de Viena sobre Derecho de los Tratados respecto de la interpretación de los mismos) la corte concluye que del texto del tratado, así como de su contexto y a la luz de su objeto y fin, no puede ser interpretado en el sentido de establecer una línea divisoria de soberanía sobre las islas más allá de la parte este de la Isla Sebatik, y por tanto, el convenio no constituye un título jurídico sobre el cual Indonesia pueda fundar sus reclamaciones sobre las islas Ligitan y Sipadan. Tal conclusión que se ve confirmada por los travaux préparatoires y la práctica posterior de las partes. Además, la corte considera que los mapas aportados por las partes no permiten hacer conclusiones respecto de la interpretación del artículo IV del convenio. ${ }^{6}$

Posteriormente, la corte analiza los títulos jurídicos basados en la sucesión por parte de Indonesia y Malasia a partir de la posesión original de los sultanes de Bulungan o de Sulu, respectivamente. Como es evidente, ambas partes disienten respecto del titular de la posesión original. Para Indonesia, el sultán de Bulungan, como soberano original, habría transmitido a Holanda el título de soberanía a través de diversos acuerdos de vasallaje incorporando sus posesiones a las indias holandesas. Al respecto, la corte considera que, si bien en los acuerdos entre el sultán y Holanda se mencionan las islas Tarakan, Nanukan y Sebatik, entre las posesiones del sultán, y se agrega "with the islets belonging there to", tal expresión sólo puede ser interpretada como referida a las pequeñas islas situadas en la vecindad inmediata de las tres islas que son mencionadas expresamente, y no, como lo pretende Indonesia, a las islas Ligitan y Sipadan situadas en una distancia de más de 40 millas náuticas. ${ }^{7}$ En conse-

6 CIJ, Case Concerning Sovereignty..., cit., nota 1, párrs. 52, 58 y 80.

7 En este punto, como recuerda Fabio Spadi, la corte sugiere un criterio cuantitativo de delimitación de los conceptos de dependencia, continuidad y contigüidad (que tanta influencia tuvieron durante el siglo XIX y que posteriormente cedieron terreno al criterio basado en el ejercicio efectivo de autoridad respecto de un territorio) basado en el presente caso en la distancia mayor a 40 
cuencia la corte no puede aceptar la posición de Indonesia como legítima heredera del título sobre las islas disputadas a partir de los acuerdos entre el sultán y los Países Bajos. ${ }^{8}$

De igual forma, la corte rechaza las pretensiones de Malasia basadas en una serie de transferencias del título originalmente sustentado por el sultán de Sulu y que habría pasado por una cadena de títulos (chain of title) a España, los Estados Unidos de América, Gran Bretaña por medio del Estado de Borneo Norte, el Reino Unido de Gran Bretaña e Irlanda del Norte y finalmente a Malasia. Lo anterior, proveería a Malasia de un título convencional sobre Ligitan y Sipadan con base en el principio de sucesión. ${ }^{9}$ La CIJ hace notar que las islas en disputa no se mencionan expresamente en ninguno de los instrumentos legales internacionales presentados por Malasia para probar las pretendidas transferencias consecutivas del título de soberanía; además de que las dos islas no fueron incluidas en la cesión del sultán de Sulu sobre todos sus derechos y poderes sobre sus posesiones en Borneo, incluyendo las islas dentro de un límite de 3 millas náuticas realizada en 1878, hecho no impugnado por las partes. En consecuencia, la corte no reconoce la pretensión de Malasia sobre la existencia de una serie ininterrumpida de transferencias de título desde la del poseedor del título original, el sultán de Sulu, en tanto no existe certeza sobre el hecho mismo de que Ligitan y Sipadan formaran parte de las posesiones del dicho sultán, así como tampoco de que algunos de los poseedores de título subsiguiente hayan tenido un título convencional sobre tales islas. La corte niega, por tanto, que Malasia haya heredado un título convencional desde su predecesor, el Reino Unido de Gran Bretaña e Irlanda del Norte. ${ }^{10}$

En este punto la corte constata que mientras ambas partes sostienen que las islas de Ligitan y Sipadan no eran terrae nullius durante el periodo en cuestión, ambas presentan títulos diametralmente opuestos y dado que no encuentra algún otro título jurídico capaz de atribuir soberanía sobre las islas en discordia pasa a estimar los argumentos de las partes con

millas náuticas como factor decisivo para determinar la no dependencia de las islas en controversia a la isla Sebatik en ausencia de otros factores relevantes. Spadi, Fabio, op. cit., nota 1, pp. 299 y ss.

8 CIJ, Case Concerning Sovereignty..., cit., nota 1, párr. 96.

9 Sobre el tema de la continuidad de tratados véase López Martín, Ana G., "La reafirmación de la regla de la continuidad en los tratados de frontera (a propósito de la sentencia del TIJ en el asunto de la controversia fronteriza Libia-Chad)", Revista Española de Derecho Internacional, Madrid, vol. XLIX, núm. 1, enero-junio de 1997, pp. 191-115.

10 CIJ, Case Concerning Sovereignty..., cit., nota 1, párr. 124. 
base a las efectividades de forma autónoma, a pesar de que las partes pretendieron que tales efectividades confirmaran sus títulos jurídicos de base convencional.

\section{ESBOZO SOBRE LAS RELACIONES ENTRE LOS TÍTULOS JURÍDICOS Y LAS EFECTIVIDADES}

La cuestión del título de la soberanía territorial ha sido largamente estudiada por la doctrina y su relevancia en la práctica internacional es evidente. ${ }^{11}$ Se suele distinguir entre el título y los modos de adquirirlo o, mejor dicho, los fundamentos del mismo. Tales fundamentos del título jurídico "son los procesos jurídicos o fácticos que el derecho internacional reconoce como capaces para la creación de un título a la soberanía territorial". ${ }^{12}$ En una controversia de atribución de soberanía sobre determinado territorio, en el caso que nos ocupa de carácter insular, lo que se valora son los títulos que enarbolan las partes en litigio y el denominado "juego de las efectividades". ${ }^{13}$ Este papel concurrente de los títulos y las efectividades se presenta de diferentes formas; ya sea que las partes estimen que su título deriva de un tratado, que el fundamento del título derive de la ocupación efectiva o del principio del uti possidetis juris, o que

11 Respecto de la noción de "título jurídico", la CIJ en su sentencia de 22 de diciembre de 1986 en el caso sobre la controversia fronteriza entre Burkina Faso y Mali, consideró que la noción de título jurídico se refiere tanto al medio de prueba susceptible de establecer un derecho, como a la fuente misma de este derecho. En palabras de la Sala: "In fact, the concept of title may also, and more generally, comprehend both any evidence which may establish the existence of a right, and the actual source of that right". Case Concerning the Frontier Dispute (Burkina Faso-Republic of Mali), 22 de diciembre de 1986, CIJ, Reports of Judgments, Advisory Opinions and Orders, 1986, p. 564, párr. 18 (www.cij-cij.org). Esto es lo que la doctrina ha denominado la doble acepción del término "título jurídico" en derecho internacional. Por una parte la causa o fundamento de un derecho (los hechos, situaciones o actos jurídicos internacionales que permiten el establecimiento de la soberanía territorial) y por el otro el documento mismo que se invoca como tal. Sobre el particular se consideraron: Distefano, Giovanni, "La notion de titre juridique et les différends territoriaux dans l'ordre international", Revue Genérale de Droit International Public, t. 100/1995/2, pp. 335 y ss; González Campos, Julio, Curso de derecho internacional público, 2a. ed., Madrid, Civitas, 2002, p. 548; López Martín, Ana Gemma, El territorio estatal en discusión: la prueba del título, Madrid, McGraw-Hill, 1999, pp. 8 y ss; Post, Harry, "International law between dominium and imperium; some reflections on the foundations of the international law on territorial acquisition", en Gill, Terry D. y Heere, Hwybo P. (eds.), op. cit., nota 1, pp. 149 y ss; Remiro Brotóns, Antonio et al., Derecho internacional, Madrid, Mc-Graw Hill, 1997, pp. 523 y ss.

12 Remiro Brotóns et al., op. cit., nota anterior, pp. 523, 535.

13 Se entiende por "efectividades" al ejercicio continuo y pacífico de funciones de Estado en determinado territorio. Cfr. López Martín, El territorio estatal..., cit., nota 11, p. XXV. 
el examen de tales títulos se haga a la luz del comportamiento ulterior de las partes, existe una relación entre el título y las efectividades que es valorada por el juez internacional.

Así lo puso de manifiesto la Sala de CIJ en el asunto de la controversia fronteriza entre Burkina Faso y Mali al relacionar el título derivado, en este caso, del principio del uti possidetis y el papel de las efectividades:

Where the act corresponds exactly to law, where effective administration is additional to the uti possidetis juris, the only role of effectivite is to confirm the exercise of the right derived from a legal title. Where the act does not correspond to the law, where the territory which is the subject of the dispute is effectively administered by a State other than the one possessing the legal title, preference should be given to the holder of the title. In the even that the effectivite does not co-exist with any legal title, it must invariably be taken into consideration. Finally, there are cases where the legal title is not capable of showing exactly the territorial expanse to which it relates. The effectivité can then play an essential role in showing how the title is interpreted in practice. ${ }^{14}$

En este sentido, se dice que las efectividades a) Pueden jugar una función confirmativa del título jurídico cuando el hecho corresponde al derecho (adecuación de una administración efectiva al uti possidetis); b) Pueden no tener valor alguno si el hecho no corresponde con el derecho (el territorio está administrado por un Estado distinto al que posee el título y, por tanto, prevalece este último); c) Puede tener una función sustitutoria si no hay título alguno; o, d) Puede jugar una función interpretativa o integradora si el título es insuficiente u oscuro. ${ }^{15}$

Para fines del presente trabajo conviene destacar el papel de las efectividades en los conflictos de atribución pues generalmente se considera

14 Case Concerning the Frontier Dispute (Burkina Faso-Republic of Mali), 22 de diciembre de 1986, Reports 1986, p. 587, párr. 63. Otro aspecto relevante de este caso es la relativización que hace la Sala de la clásica distinción entre "conflictos de delimitación" y "conflictos de atribución" al considerar que entre ellas sólo hay una distinción de grado, lo que a su vez relativiza el papel del uti possidetis y de las efectividades en tales conflictos. Al respecto, Sánchez Rodríguez, Luis Ignacio, "Uti possidetis: la reactualización jurisprudencial de un viejo principio (a propósito de la sentencia del TIJ [Sala] en el asunto Burkina Fasso-República de Mali)", Revista Española de Derecho Internacional, Madrid, vol. XL, 1988, núm. 2, julio-diciembre de 1988, pp. 122; González Campos et al., op. cit., nota 11, p. 549; López Martín, El territorio estatal..., cit., nota 11, p. 53.

15 Cfr. López Martín, El territorio estatal..., cit., nota 11, p. 54; González Campos et al., op. cit., nota 11, p. 557; Remiro Brotóns et al., op. cit., nota 11, p. 535 
que en estos conflictos juegan un papel primordial. Aunque la propia sala de la CIJ en el asunto de la controversia fronteriza entre Burkina Faso y Mali estableció solamente una distinción de grado entre controversias de delimitación y de atribución y, por lo tanto, también relativizó la importancia del título y de las efectividades en tales controversias, el presente caso de Indonesia-Malasia nos muestra el juego que estas últimas tienen en ausencia de otros títulos jurídicos, y es claro ejemplo de cómo en estos supuestos, en palabras de Giovanni Distefano, "la décision juridictionnelle... permettra d'elever l'effectivité au rang de titre". ${ }^{16}$

En efecto, es la función sustitutoria de las efectividades la que se presenta en los supuesto en que no coexisten con ningún otro título jurídico, tal como lo señaló la CIJ en el asunto sobre la controversia fronteriza (Burkina Faso-Mali) y lo reiteró en el caso de la controversia fronteriza terrestre, insular y marítima entre el Salvador y Honduras. ${ }^{17}$ En este último caso, si bien las partes acordaron la aplicación del principio del uti possidetis iuris como derecho aplicable en todo el contencioso, la Sala de la CIJ consideró inviable su aplicación respecto de ciertas islas del Golfo de Fonseca dada las lagunas y ambigüedades de las evidencias aportadas por las partes y estimó necesario referirse a las efectividades post-coloniales aunadas a ciertas conductas que pudieran considerarse como aquiescencia de tales efectividades.

Sobre el particular la sala consideró:

347. Thus it was not until a number of years after the independence of the two States that the question of the appurtenance of the islands of the Gulf to the one or the other became of significant import. What then occurred appears to the Chamber to be highly material. The islands were not terra nullius, and in legal theory each island already appertained to one of the three States surrounding the Gulf as heir to the appropriate part of the Spanish colonial possessions, so that acquisition of territory by occupation was not possible; but the effective possession by one of the Gulf States of any island of the Gulf could constitute an effectivity, though a post-colonial one, throwing light on the contemporary appreciation of the legal situation. Possession backed by the exercise of sovereignty may be taken as evidence confirming the uti possidetis juris title. The Chamber does not find it necessary to decide whether such possession could be recognized

16 Distefano, Giovanni, op. cit., nota 11, p. 362.

17 Case Concerning the Land, Island and Maritime Frontier Dispute (El Salvador-Honduras: Nicaragua intervening), 11 de septiembre de 1992. CIJ, Reports 1992, p. 398, parr. 61. 
even in contradiction of such title, but in the case of the islands, where the historical material of colonial times is confused and contradictory, and the accession to independence was not immediately followed by unambiguous acts of sovereignty, this is practically the only way in which the uti possidetis juris could find formal expression so as to be judicially recognized and determined. ${ }^{18}$

Lo anterior, significa, en nuestro concepto, un distanciamiento de las efectividades respecto de la concepción clásica de la ocupación territorial como fundamento del título de soberanía territorial, en tanto que para considerar que la ocupación ha cristalizado en un título jurídico definitivo de adquisición de la soberanía territorial, es preciso que en el momento en que se produzca el hecho físico de la ocupación, el territorio sea terra nullius y que la ocupación sea efectiva y en carácter de soberanía (corpus y animus). ${ }^{19}$ En materia de efectividades, cuando éstas asumen una función sustitutoria, el hecho de que el territorio sea considerado terra nullius deja de ser una condición previa para la operatividad de la ocupación efectiva siendo necesario solamente la constatación del corpus y el animus occupandi. Como veremos a continuación, éste fue el supuesto de la controversia entre Indonesia y Malasia respecto de la soberanía islas Ligitan y Sipadan en donde ambas sostuvieron que las islas no eran terrae nullius.

18 Ibidem, p. 219. Sobre la función sustitutoria de las efectividades: López Martín, A., El territorio estatal..., cit., nota 11.

19 La condición previa se refiere a que el territorio ocupado sea en ese momento terra nullius (como lo consideró el árbitro en el asunto de la isla Clipperton y lo reiteró la CIJ en su opinión consultiva sobre el Sahara occidental) y la doble condición posterior al hecho físico de la ocupación consistente en que el Estado manifieste un animus occupandi sobre el territorio y además una ocupación efectiva (así se expresa, respectivamente, en el asunto de la isla Clipperton, en el asunto de Groenlandia oriental del Tribunal Permanente de Justicia Internacional, en el asunto arbitral de la Isla de Palmas, en la sentencia arbitral del asunto de la soberanía territorial (Eritrea-Yemen); y así lo ha reiterado la CIJ en su opinión sobre el Sahara occidental y en los casos de los Minquiers y Ecréthos; de la isla Kasikili-Sedudu; de la delimitación marítima y cuestiones territoriales entre Qatar y Bahrein. De esta forma, "la ocupación se configura como un modo originario de adquirir la soberanía territorial sobre territorio nullius, con voluntad del Estado ocupante de convertirse en soberano, manifestada a través del ejercicio de sus funciones propias, con actos de soberanía frente a otros Estados, durante un plazo razonable de tiempo, a cuyo fin el título inicial de simple ocupación física deviene un título jurídico definitivo y oponible erga omnes". Cfr. González Campos et al., op. cit., nota 11, pp. 550-553. 


\section{BREVE REFERENCIA A LAS EFECTIVIDADES Y LA CUESTIÓN DE LA FECHA CRÍTICA}

Una vez analizados y descartados los títulos alegados por las partes, la corte consideró las evidencias presentadas por las mismas con respecto a las efectividades tendentes a resolver la cuestión de la soberanía sobre las islas Ligitan y Sipadan, para ello recordó su jurisprudencia constante en el sentido de que en el supuesto de que las efectividades no coexistan con ningún título jurídico, deben invariablemente ser tomadas en cuenta. ${ }^{20}$

En este sentido, ya se ha dicho que existe un rol concurrente de los títulos jurídicos y de las efectividades que puede adoptar diferentes formas y que en ausencia del primero, la importancia jurídica de las efectividades, cuando éstas asumen una función sustitutoria, se vuelve imprescindible. El principio fundamental de la efectividad hace que del ejercicio efectivo de funciones estatales en un territorio determinado nazca la soberanía sobre el mismo. Tal principio se encuentra limitado por el de la relatividad de las efectividades que "modula y flexibiliza" el principio anterior, en el sentido de que la intensidad y las características de las funciones estatales han de ser apreciadas sobre la base de las circunstancias del caso concreto. ${ }^{21}$ Por ello, la prueba de la ocupación efectiva, en la constatación del corpus y el animus occupandi, conlleva la revisión de los actos llevados a cabo por los Estados en la controversia de forma independiente y distinta, tal como lo destaca la CIJ en el presente caso.

Si se entiende por ocupación efectiva el ejercicio exclusivo, continuado, pacífico y prolongado de las funciones estatales en un territorio determinado, las efectividades constituyen la prueba de dicho comportamiento de las autoridades estatales y por tanto del ejercicio efectivo de atribuciones estatales en el territorio en disputa. Para ese efecto, la cues-

20 "126... The Court recalls that it has already ruled in a number of cases on the legal relationship between 'effectivités' and title. The relevant passage for the present case can be found in the Judgment in the Frontier Dispute (Burkina Faso-Republic of Mali) case, where the Chamber of the Court stated after having said that "a distinction must be drawn among several eventualities": "[i]n the event that the effectivite does not co-exist with any legal title, it must invariably be taken into consideration" (ICJ Reports 1986, p. 587, parr. 63; see also Territorial Dispute (Libyan Arab Jamahiriya-Chad), ICJ Reports 1994, p. 38, párrs. 75-76; Land and Maritime Boundary between Cameroon and Nigeria (Cameroon v. Nigeria: Equatorial Guinea intervening), Judgment, Merits, ICJ Reports 2002, párr. 68).

21 Pastor Ridruejo, José Antonio, Curso de derecho internacional público y organizaciones internacionales, 8a. ed., Madrid, Tecnos, 2002, p. 324. 
tión del animus occupandi debe constatarse principalmente en la consideración de las actividades desplegadas efectivamente en el territorio como hechas a título de soberanía (á titre de souverain) por agentes estatales o con su autorización o ratificación y no por personas no autorizadas sin que exista un consentimiento de otro Estado que reivindique para sí la soberanía territorial; además, la naturaleza predominante de la actividad debe desarrollarse en el contexto del ejercicio de las atribuciones estatales. $^{22}$ Tales consideraciones deben hacerse en el contexto general de las circunstancias propias del territorio en disputa y considerando el momento de la fecha crítica en que la controversia se manifiesta.

En el caso de las islas Ligitan y Sipadan, el análisis de las evidencias presentadas por las partes en tanto efectividades tendentes a acreditar la ocupación efectiva como título jurídico de las islas en discordia debió considerar la cuestión de la fecha crítica. Sobre este aspecto también la sentencia representa una confirmación de la jurisprudencia internacional en la materia.

La cuestión de la fecha crítica en las controversias territoriales ha sido ampliamente estudiada por la doctrina y existe una firme y reiterada jurisprudencia internacional en la materia. ${ }^{23}$ Como recuerda Marcelo Kohen, ${ }^{24}$ "la doctrina se refiere en general a la fecha crítica como el momento a partir del cual el comportamiento posterior de las partes en una controversia no puede ser tomado en consideración para la solución de la misma". Aunque de manera excepcional pueden ser tenidos en cuenta los hechos posteriores a la fecha crítica, como medios probatorios que permitan corroborar la decisión del tribunal.

En este sentido, la fecha crítica ha sido considerada como:

22 Cfr. Brownlie, Ian, Principles of Public International Law, 5a. ed., Oxford University Press, 1999, p. 138.

23 Sobre este tema: González Campos et al., op. cit., nota 11, pp. 553 y ss.; Kohen, Marcelo, "La fecha crítica y la cuestión de las Islas Malvinas", Revista Española de Derecho Internacional, Madrid, vol. XLVI, núm. 1, 1994, pp. 7-37; López Martín, A., El territorio estatal.., cit., nota 11, pp. 114 y ss.; Sánchez Rodríguez, Luis Ignacio, "Fecha crítica, contenciosos territoriales y jurisprudencia internacional", en varios autores, Hacia un nuevo orden internacional y europeo. Estudios en homenaje al profesor don Manuel Díez de Velasco, Madrid, Tecnos, 1993, pp. 661-687; e $i d$. ." "El problema de la fecha crítica en los litigios relativos a la atribución de la soberanía territorial del Estado", Anuario de Derecho Internacional IV, Bogotá, Universidad de Pamplona, 1977-1978, pp. 53-88.

24 Kohen, M., op. cit., nota anterior, p. 8. 
La regla de naturaleza procesal y sustantiva, de origen jurisprudencial, que en las controversias internacionales de carácter territorial sirve para determinar el momento en que se ha consolidado un título histórico, o para precisar el tratado que resume definitivamente la voluntad de las partes de resolver una cuestión de límites. La fase temporal inmediatamente anterior se denomina periodo crítico, y es de importancia esencial para valorar la situación jurídica de las partes. Excepcionalmente pueden ser tenidos en cuenta los hechos posteriores a la fecha crítica, a los únicos fines de corroborar la decisión del tribunal. ${ }^{25}$

Sobre el particular, tanto la práctica arbitral como jurisdiccional es abundante. ${ }^{26}$ Sin ser exhaustivos y sólo como referente cabe mencionar algunos casos de determinación de la fecha crítica y sus efectos con el objeto de precisar el momento en que, desde el punto de vista de las efectividades, debe acreditarse el ejercicio efectivo y pacífico de las funciones estatales en determinado territorio en discusión, en particular cuando, como en el caso que comentamos, el asunto se remonta siglos atrás.

En el asunto de la Isla de Palmas, el árbitro Max Huber, después de determinar que la fecha crítica era el 10 de diciembre de 1898, fecha de celebración del Tratado de París entre España y Estados Unidos de América por el que se ceden ciertos territorios a este último país, señaló:

It is not necessary that the display of sovereignty should go back to a very far distant period. It may suffice that such display existed in 1898, and had already existed as continuous and peaceful before that date long enough to enable any Power who might have considered herself as possessing sovereignty over the island, or having a claim to sovereignty, to have, according to local conditions, a reasonable possibility for ascertaining the existence of a state of things contrary to her real or alleged rights.

25 González Campos et al., op. cit., nota 11, p. 556. Para los propósitos de esta exposición no consideramos necesario entrar al debate sobre la naturaleza sustantiva, procesa o mixta de la fecha crítica, y sólo apuntamos que para algunos autores "la fecha crítica constituye una categoría jurídica de naturaleza mixta, sustantiva y procesal, que cumple una doble función: en los contenciosos relativos a la adquisición el territorio por un título jurídico, la fecha crítica permite determinar el momento a partir del cual el comportamiento de las partes ha consolidado definitivamente un título provisional, siendo irrelevantes los actos de las partes posteriores a ese momento. En los contenciosos que se refieren a la delimitación del territorio, la fecha crítica señala el tratado que resume definitivamente la voluntad de las partes de solucionar una cuestión de límites" (Sánchez Rodríguez, L., "El problema de la fecha crítica...", op. cit., nota 23, p. 87). Mientras que para otros se trata de una regla de carácter técnico, "un instrumento que permite la aplicación de las reglas de fondo a una controversia o a una reivindicación en particular". Kohen, M., op. cit., nota 23, p. 10.

26 Cfr. Sánchez Rodríguez, L., "El problema de la fecha crítica...", op. cit., nota 23, pp. 67 y ss. 
It is not necessary that the display of sovereignty should be established as having begun at a precise epoch; it suffices that it had existed at the critical period preceding the year 1898. It is quite natural that the establishment of sovereignty may be the outcome of a slow evolution of a progressive intensification of State control. ${ }^{27}$

Asimismo, la Corte Permanente de Justicia Internacional en el asunto del estatuto jurídico de Groenlandia Oriental consideró que no era necesario que la soberanía danesa sobre Groenlandia haya existido de forma absoluta durante un largo periodo de tiempo sino que bastaba con que dicha soberanía existiera al momento de la ocupación de Noruega; siendo la fecha crítica la de 10 de julio de 1931, fecha de la ocupación noruega.

Respecto de los hechos posteriores a la fecha crítica, la CIJ en el caso de los grupos de islotes y rocas de los Minquiers y Ecrehos, frente a las consideraciones de las partes (Reino Unido y Francia) respecto de la fecha crítica, estimó:

A dispute as to sovereignty over the groups did not arise before the years 1886 and 1888, when France for first time claimed sovereignty over the Ecrehos and the Minquiers respectively. But in view of the special circumstances of the present case, subsequent acts should also be considered by the Court, unless the measure in question was taken with a view to improving the legal position of the Party concerned. ${ }^{28}$

Ahora bien, si, como señala Marcelo Kohen, en lo que respecta a la adquisición de la soberanía territorial propiamente dicha, "la fecha de nacimiento de una controversia será aquella en la que una parte reivindica la soberanía sobre un territorio y la otra rechaza tal pretensión", conviene determinar el momento que debe considerarse para determinar la fecha crítica. ${ }^{29}$ Dentro de las hipótesis que se consideran para determinar la fecha crítica, ${ }^{30}$ hay que resaltar aquella que considera como fecha crítica la

27 Island of Palmas (or Milangas) (Netherlans-United States of America), 4-IV-1928. Texto disponible en Coussirat, Vicent y Eisemann, Pierre (eds.), Repertory of International Arbitral Jurisprudence, Martinus Nijhoff Publishers, t. II, vol. II, 1919-1945, pp. 76 y 77.

28 CIJ, The Minquiers and Ecrehos Case (France-United Kingdom, Judgment of november 17th, 1953, Reports 1953), p. 59.

29 Kohen, M., op. cit., nota 23, p. 20.

30 Entre las diferentes fechas, se consideran: la fecha del comienzo de la controversia; la fecha de la primera reivindicación del Estado demandante; la fecha en que cristaliza la controversia; fecha en que una parte toma medidas para iniciar un procedimiento de arreglo de controversias; 
fecha en que cristaliza la controversia de forma definitiva entre las partes respecto de la soberanía sobre el territorio. Al respecto, se considera que la cristalización "es el momento en el cual toman cuerpo las posiciones respectivas de las partes; es el instante en el que pueden identificarse los hechos o títulos que cada una de las partes alegan, así como la extensión territorial de sus reclamos". ${ }^{31}$

En el caso que se comenta entre Indonesia y Malasia, el tribunal determina como fecha crítica 1969 en tanto que: "31. [...] The present dispute crystallized in 1969 in the context of discussions concerning the delimitation of the respective continental shelves of the two State".

Sobre este punto en la sentencia se destaca que durante las negociaciones de 1969, Malasia reivindicó su soberanía sobre las islas Ligitan y Sipadan, con la consiguiente oposición de Indonesia. Por tales motivos:

128. [...] According to Indonesia, it was thus at that time that the "critical date" arose in the present dispute. It contends that the two Parties undertook, in an exchange of letters of 22 September 1969, to refrain from any action which might alter the status quo in respect of the disputed islands. It asserts that from 1969 the respective claims of the Parties therefore find themselves "legally neutralized", and that, for this reason, their subsequent statements or actions are not relevant to the present proceedings.

A pesar de ello, en opinión de Indonesia, Malasia, desde 1979 en adelante, realizó una serie de medidas unilaterales incompatibles con la situación existente en 1969, a lo que Indonesia supuestamente protestó constantemente. Por su parte, Malasia estima que la corte debe considerar aquellas actividades que iniciaron antes de la fecha crítica y que continuaron después de ella y respecto de las cuales se debieron tomar algunas medidas específicas. Al respecto se lee en la sentencia:

129. With respect to the critical date, Malaysia begins by asserting that prior to the 1969 discussions on the delimitation of the continental shelves of the Parties, neither Indonesia nor its predecessors had expressed any interest in or claim to these islands. It however emphasizes the importance

fecha en que tales procedimientos se utilizan y se ponen en práctica; fecha en que, fracasados otros medios, se somete el asunto al órgano jurisdiccional. Cfr. Sánchez Rodríguez, L., "Fecha crítica, contenciosos...", op. cit., nota 23, p. 670; López Martín, A. El territorio estatal..., cit., nota 11, pp. 116 y 117.

31 Kohen, M., op. cit., nota 23, p. 22. 
of the critical date, not so much in relation to the admissibility of evidence but rather to "the weight to be given to it". Malaysia therefore asserts that a tribunal may always take into account post-critical date activity if the party submitting it shows that the activity in question started at a time prior to the critical date and simply continued thereafter. As for scuba-diving activities on Sipadan, Malaysia observes that the tourist trade, generated by this sport, emerged from the time when it became popular, and that it had itself accepted the responsibilities of sovereignty to ensure the protection of the island's environment as well as to meet the basic needs of the visitors.

Con base en lo anterior, la corte enfatiza y puntualiza el efecto que tienen los hechos posteriores a la fecha crítica, así como aquellos hechos de carácter continuado que no tienen por objeto mejorar la posición de las partes en el litigio:

135. The Court further observes that it cannot take into consideration acts having taken place after the date on which the dispute between the Parties crystallized unless such acts are a normal continuation of prior acts and are not undertaken for the purpose of improving the legal position of the Party which relies on them (see the Arbitral Award in the Palena case, 38 International Law Reports (ILR), pp. 79-80).

De esta forma, la corte pasa a considerar, en primer lugar "the effectivités which date from the period before 1969, the year in which the Parties asserted conflicting claims to Ligitan and Sipadan", en el entendido de que sólo considerara aquellos actos que, con los matices que veremos a continuación, expresen un significativo ejercicio de autoridad y que se refieren específicamente a las islas en disputa. En palabras de la corte:

136. The Court finally observes that it can only consider those acts as constituting a relevant display of authority which leave no doubt as to their specific reference to the islands in dispute as such. Regulations or administrative acts of a general nature can therefore be taken as effectivités with regard to Ligitan and Sipadan only if it is clear from their terms or their effects that they pertained to these two islands. 


\section{EL JUEGO DE LAS EFECTIVIDADES EN ACCIÓN}

Como en otros casos de controversia territorial, el asunto sobre la soberanía de las islas Ligitan y Sipadan, dadas las particularidades geográficas de las islas, su pequeña extensión y su escasa importancia económica, planteó la consideración del principio de relatividad del ejercicio de las efectividades. ${ }^{32} \mathrm{Al}$ respecto, la corte retoma la jurisprudencia internacional en la materia y precisa: "In particular in the case of very small islands which are uninhabited or not permanently inhabited like Ligitan and Sipadan, which have been of little economic importance (at least until recently) effectivités will indeed generally be scarce" (párr. 134).

En consecuencia, la CIJ recuerda los elementos necesarios de toda ocupación territorial (corpus y animus). Para ello, se remite al asunto del Estatuto Jurídico de Groenlandia Oriental, resuelto por la Corte Permanente de Justicia Internacional mediante sentencia de 5 de abril de 1953, en la cual el tribunal, entre otras cosas, señaló: "A claim to sovereignty based not upon some particular act or title such as treaty of cession but merely upon continued display of authority, involves two elements each of which must be shown to exist: the intention and will to act as sovereign, and some actual exercise or display of such authority". ${ }^{33}$

La Corte Permanente continúa:

It is impossible to read the records of the decisions in cases as to territorial sovereignty without observing that in many cases the tribunal has been satisfied with very little in the way of the actual exercise of sovereign rights, provided that the other State could not make out a superior claim. This is

32 Tal criterio fue reconocido ya en el arbitraje de la Isla de Palmas (1928) entre Holanda y Estados Unidos de América, en el cual el árbitro único, Huber, estimó que "the continuous and paeceful display of territorial sovereignty is as good as a title" y puntualizó que tales manifestaciones de soberanía pueden adoptar diferentes formas de acuerdo con las condiciones de tiempo y lugar en que se ejerzan. En palabras del árbitro Huber: "Manifestations of territorial sovereignty assume, it is true, different forms, according to conditions of time and place. Although continuous in principle, sovereignty cannot be exercised in fact at every moment on every point of a territory. The intermittence and discontinuity compatible with the maintenance of the right necessarily differ according as inhabited or uninhabited regions are involved, or regions enclosed within territories in which sovereignty is incontestably displayed or again regions accessible from, for instance, the high seas". Island of Palmas (or Milangas) (Netherlans-United States of America), 4-IV-1928, Repertory of International..., cit., nota 27, p. 80.

33 CPJI, Legal Status of Eastern Greenland, 5 de abril de 1933, Judgments, Orders and Advisory Opinions, vol. 2, núms. 49-62, 1932-1934, Kraus Reprint, 1970, p. 45. 
particularly true in the case of claims to sovereignty over areas in thinly populated or unsettled countries. ${ }^{34}$

Sobre el particular, y aunque la corte no se refiere a ella en su sentencia, resulta relevante la sentencia arbitral de 9 de octubre de 1998 emitida en el asunto de la soberanía territorial (Eritrea-Yemen), en la cual se destacó:

239. The factual evidence of effectivités presented to the Tribunal by both parties is voluminous in quantity but is sparse in useful content. This is doubtless owing to the inhospitability of the Islands themselves and the relative meagreness of their human history. The modern international law of the acquisition (or attribution) of territory generally requires that there be: an intentional display of power and authority over the territory, by the exercise of jurisdiction and state functions, on a continuous and peaceful basis. The latter two criteria are tempered to suit the nature of the territory and the size of its population, if any. ${ }^{35}$

En este contexto es que el CIJ analiza las efectividades hechas valer por las partes. Al respecto hay que recordar que aunque las partes hayan alegado ciertas efectividades con el objeto de confirmar sus pretendidos títulos convencionales, la corte va a considerar las efectividades en su función sustitutoria, de manera independiente y separada de los otros títulos jurídicos reivindicados.

En cuanto a las efectividades alegadas por Indonesia en la sentencia se señala: "The Court concludes that the activities relied upon by Indonesia do not constitute acts à titre de souverain reflecting the intention and will to act in that capacity". ${ }^{36}$ Para llegar a esa conclusión la corte empieza por aclarar que ninguna de ellas tiene carácter legislativo o regulador. Respecto de la Ley núm. 4 que define las líneas de base de las aguas archipelágicas indonesas, la corte precisa que ésta no hace referencia a las islas Ligitan y Sipadan como parámetros para medir las líneas de base de

34 Ibidem, p. 46.

35 Eritrea-Yemen Arbitration. Award of the Arbitral Tribunal in the First Stage (Territorial Sovereignty and Scope of the Dispute), 9 de octubre de 1998. Disponible en http://www.pca-cpa.org/ENGLISH/RPC/EY/2ch3ER-YE.htm. Sobre la importancia de esta sentencia arbitral véase: Márques Antunes, Nuno Sergio, "The Eritrea-Yemen arbitration: First stage-the law of title to territory re-averred", International and Comparative Law Quarterly, vol. 48, abril de 1999, parte 2, pp. 362-386.

36 CIJ, Case concerning sovereignty..., cit., nota 1, párr. 141. 
las aguas archipelágicas o el mar territorial, y por tanto no puede considerarse como ejercicio de soberanía. De igual forma, de la continua presencia de la armada holandesa primero y de la indonesa después no puede deducirse que las autoridades navales concernidas consideren las islas en disputa y sus aguas territoriales como pertenecientes a la soberanía de Holanda o Indonesia. Finalmente, respecto de la presencia de pescadores nacionales indonesios en las aguas que rodean las islas, la corte aduce que las actividades de personas privadas o particulares no pueden ser consideradas como actos de soberanía si no son realizadas sobre la base de regulaciones oficiales o bajo autoridad gubernamental. En palabras de la corte "that activities by private persons cannot be seen as effectivités if they do not take place on the basis of official regulations or under governmental authority". ${ }^{37}$

Por su parte, Malasia alegó, entre otras cosas, el ejercicio de efectividades relativas a ciertas medidas adoptadas por las autoridades de la antigua colonia de Borneo del Norte para regular y controlar la recolección de los huevos de tortuga, la construcción de un santuario de aves, así como la construcción por las mismas autoridades y el mantenimiento por Malasia hasta la actualidad de faros marítimos construidos en 1963 y 1964 en las islas.

Al respecto, la corte consideró que las medidas de regulación y control de la recolección de huevos de tortuga así como el establecimiento del santuario para las aves, constituyen medidas reglamentarias y administrativas que implican el ejercicio de autoridad específicamente sobre los territorios en discusión. ${ }^{38}$ En relación con el establecimiento y mantenimiento de faros y ayudas a la navegación, la corte precisó que, si bien la construcción y operación de faros y ayudas a la navegación no se han considerado como manifestaciones de autoridad estatal, tales actividades pueden ser legalmente relevantes en el caso de islas pequeñas y considerarse suficientes para probar el ejercicio de soberanía sobre el territorio. ${ }^{39}$

37 CIJ, Case concerning sovereignty..., cit., nota 1, párr. 140. Criterio similar respecto de la actividad de particulares siguió respecto de los Masubia de Caprivi Oriental en el caso relativo a la Isla Kasikili-Sedudu (Botswana-Namibia) de 13 de diciembre de 1999.

38 La sentencia señala: "145. The Court is of the opinion that both the measures taken to regulate and control the collecting of turtle eggs and the establishment of a bird reserve must be seen as regulatory and administrative assertions of authority over territory which is specified by name".

39 Al respecto se dice en la sentencia: "147. The Court observes that the construction and operation of lighthouses and navigational aids are not normally considered manifestations of State authority (Minquiers and Ecrehos, Judgment, ICJ Reports 1953, p. 71). The Court, however, recalls 
Finalmente, la corte concluye que si bien las actividades realizadas por Malasia, tanto en su propio nombre como en su carácter de sucesora de Gran Bretaña, son modestas en número, son diversas en su naturaleza e incluyen el ejercicio de actos legislativos, administrativos y cuasi-judiciales, durante un periodo considerable de tiempo que muestra un patrón de ejercicio de funciones estatales sobre las dos islas. ${ }^{40}$ Además, la corte considera que durante el periodo de tales actuaciones ni Indonesia ni su predecesor Holanda expresaron desacuerdo alguno, lo que no es usual en atención a las actividades realizadas, en palabras de la corte:

In this regard, the Court notes that in 1962 and 1963 the Indonesian authorities did not even remind the authorities of the colony of North Borneo, or Malaysia after its independence, that the construction of the lighthouses at those times had taken place on territory which they considered Indonesian; even if they regarded these lighthouses as merely destined for safe navigation in an area which was of particular importance for navigation in the waters off North Borneo, such behaviour is unusual.

Finalmente, dadas las circunstancias del caso, en especial en atención a las evidencias presentadas por las partes "the Court concludes that Malaysia has title to Ligitan and Sipadan on the basis of the effectivités referred to above". Con ello la corte confirma la función sustitutoria de las efectividades en las controversias de atribución territorial y particularmente su relevancia tratándose de territorios pequeños, poco poblados o inhabitados.

that in its Judgment in the case concerning Maritime Delimitation and Territorial Questions between Qatar and Bahrain (Qatar v. Bahrain) it stated as follows: 'Certain types of activities invoked by Bahrain such as the drilling of artesian wells would, taken by themselves, be considered controversial as acts performed à titre de souverain. The construction of navigational aids, on the other hand, can be legally relevant in the case of very small islands. In the present case, taking into account the size of Qit'at Jaradah, the activities carried out by Bahrain on that island must be considered sufficient to support Bahrain's claim that it has sovereignty over it'. (Judgment, Merits, ICJ Reports 2001, parágrafo 197)". The Court is of the view that the same considerations apply in the present case".

40 La sentencia considera: "the activities relied upon by Malaysia, both in its own name and as successor State of Great Britain, are modest in number but that they are diverse in character and include legislative, administrative and quasi-judicial acts. They cover a considerable period of time and show a pattern revealing an intention to exercise State functions in respect of the two islands in the context of the administration of a wider range of islands" (párr. 148). 


\section{COMENTARIO FINAL}

El caso concerniente a la soberanía sobre Pulau Ligitan y Pulau Sipadan entre Indonesia y Malasia se presenta como una confirmación de los criterios sostenidos por el tribunal respecto del papel de las efectividades en las controversias de atribución territorial. Por esto, es un buen ejemplo del estado actual de la jurisprudencia en ese campo y expresa claramente la función sustitutoria de las efectividades a falta de otros títulos jurídicos. Sobre este punto, nos parece una sentencia particularmente reveladora en tanto considera a las efectividades de manera independiente a los otros títulos argumentos.

Otro aspecto relevante del caso es la poca diligencia manifestada por las partes para acreditar sus pretensiones, lo que revela quizá los intereses económicos y políticos que juegan tras ciertos contenciosos territoriales, particularmente con aquellos donde están en juego importantes yacimientos de hidrocarburos. ${ }^{41}$ Sobre este punto, cabe destacar la declaración que el juez Oda adjunta a la sentencia en donde, entre otras cosas, señala:

I voted in favour of the Judgment, in which the Court finds that "sovereignty over Pulau Ligitan and Pulau Sipadan belongs to Malaysia"... The present case is a rather "weak" one in that neither Party has made a strong showing in support of its claim to title to the islands on any basis. While Malaysia has made a more persuasive case on the basis of effectivités, its arguments are still not very strong in absolute terms. The Court, however, has been requested to choose between the two Parties in adjudging "whether sovereignty over [the two islands] belongs to... Indonesia or to Malaysia"... and, given that choice, the Court has come to a reasonable decision.

Desde esta perspectiva, las efectividades jugaron a favor de las partes y la CIJ actuó de alguna forma en suplencia de la deficiencia de sus pretensiones. De cualquier manera, la sentencia es clara muestra del papel de las efectividades en los conflictos territoriales en el derecho internacional actual.

41 En palabras de Marcelo Kohen: "Le pétrole est à l'origine des frontières un peu partout dans le monde", id., "Les questions territoriales dans l'arret de la CIJ du 16 de mars 2001 en l'affaire Qatar c. Bahreïn", Revue Générale de Droit International Public, t. 106/2002/2, p. 296. 
Finalmente, otra cuestión significativa, que también destaca en su declaración el juez Oda, es que si bien la controversia surge en el contexto de la delimitación marítima de las fronteras entre Indonesia y Malasia, lo cierto es que el caso planteado y resuelto por la corte es respecto de la soberanía sobre las islas en litigio y no sobre la delimitación de la frontera y las zonas marítimas, tales cuestiones son distintas y por tanto la atribución de soberanía no implica un pronunciamiento sobre dicha delimitación. Lo anterior es significativo si se tiene en cuenta las importantes reservas de petróleo en la zona; sin embargo, como lo expresa el juez Oda, la sentencia de la corte si bien atribuye la soberanía sobre las islas a Malasia, esto no tiene necesariamente efectos directos sobre la delimitación de la plataforma continental entre los dos Estados sujeta a discusión desde finales de los años sesenta del siglo XX, pues tal delimitación está sujeta a los principios establecidos en el artículo 83 de la Convención de Naciones Unidas sobre Derecho del Mar de 1982, que se refiere a la necesidad de un acuerdo entre las partes con el objeto de llegar a una "solución equitativa", aunque es innegable la influencia que la atribución de soberanía sobre pequeñas islas tiene en los procesos de delimitación de la plataforma continental.

En última instancia, que tales controversias se sometan a la decisión final de la Corte Internacional de Justicia refuerza el papel de dicha jurisdicción en general, y en las relaciones internacionales asiáticas, en particular, donde las controversias territoriales no suelen llevarse al Tribunal de La Haya. ${ }^{42}$ En efecto, el caso concerniente a la soberanía sobre Pulau Ligitan y Pulau Sipadan entre Indonesia y Malasia es el primer caso asiático llevado a la corte por compromiso o acuerdo especial (dado que ninguna de las partes ha hecho una declaración de aceptación general de la competencia contenciosa de la corte) y el primer diferendo territorial en

$42 \mathrm{Al}$ respecto, resulta pertinente considerar las reticencias de los países del sudeste asiático en someter las controversias jurídicas a órganos como la Corte Internacional de Justicia. Algunas de las razones pueden encontrarse en argumentos como el siguiente: "The ICJ is based entirely on western legal concepts, and given the importance to Asian states of asserting their post-colonial independence it seems very strange that they should turn westwards for the resolution of a bilateral dispute. When bilateral negotiations were unable to succeed the natural thing to do may have been to refer the dispute to the High Council of the Association of Southeast Asian Nations (ASEAN). The ASEAN High Council itself would not currently be a good mechanism for the resolution of such disputes because each member has its own political agenda, and equitable results are therefore unlikely". Cfr. Dowling, James, "The Significance of Ligitan and Sipadan: Indonesia vs Malaysia 1998-2002”, International Boundary Dispute Resolution, AR/5091, King's College London, 24 de marzo de 2004, p. 8. 
la región menos institucionalizada del planeta, después del caso sobre el Templo de Préah Vihéar entre Camboya y Tailandia de 1960, siendo además, como señala Delphine Perrin, una contribución notable a la jurisprudencia internacional respecto del papel de las efectividades en los conflictos de atribución a falta de otro título territorial. ${ }^{43}$ Este cambio de actitud de ciertos países del sudeste asiático se confirma recientemente con el sometimiento a la CIJ de un acuerdo especial entre Malasia y Singapur para que determine a cual de las partes corresponde la soberanía territorial sobre las islas Pedra Branca-Pulau Batu Puteh, Middle Rocks y South Ledge. ${ }^{44}$ En esta nueva controversia, el caso sobre las islas Pulau Ligitan y Pulau Sipadan entre Indonesia y Malasia constituye un precedente fundamental a tener en consideración.

43 Cfr. Perrin, D., op. cit., nota 1, pp. 322 y 323.

44 CIJ, Sovereignty over Pedra Branca/Pulau Batu Puteh, Middle Rocks and South Ledge (Malaysia-Singapore), www.icj-cij.org. 